\title{
EPIDEMIOLOGÍA DE CARDIOPATÍAS CONGÉNITAS EN UN HOSPITAL DE TERCER NIVEL, SANTO DOMINGO NORTE, REPÚBLICA DOMINICANA
}

\section{Epidemiology of congenital heart disease in a third level hospital, Santo Domingo Norte, Dominican Republic}

\section{Demian A. Herrera Morbana, Manuel Colomé-Hidalgo ${ }^{b}$, Rayneida Méndez Núñezc, Zoila L. Torres ${ }^{d}$, Stephania Cossetye Jiménez Henríquez ${ }^{f}$, Tatiana Alcántara Tiburciog y Yessi P. Alcántarah}

Recibido: 11 de febrero, $2020 \bullet$ Aprobado: 30 de marzo, 2020

Cómo citar: Herrera Morban DA, Colomé-Hidalgo M, Méndez Núñez R, Torres ZL, Cossety S, Alcántara Tiburcio T, Alcántara YP. Epidemiología de cardiopatías congénitas en un hospital de tercer nivel, Santo Domingo Norte, República Dominicana. cysa [Internet]. 17 de junio de 2020 [citado 17 de junio de 2020];4(2):37-44. Disponible en: https://revistas.intec.edu.do/index.php/cisa/article/ view/1765

\section{Resumen}

Introducción: las cardiopatías congénitas (CC) son un colectivo de patologías donde la estructura o morfología del corazón se ve afectada, es decir, se produce un trastorno en la función del órgano, originado durante la formación o desarrollo cardiovascular embrionario. Las CC representan la malformación congénita más frecuente en niños a nivel mundial, con una prevalencia de 4-14 por 1000 nacidos vivos, destacándose un aumento en los países asiáticos en $13-16 / 1000$ nacidos vivos. ${ }^{1-4,6}$

Material y Métodos: se realizó un estudio descriptivo y transversal, con recolección de datos retrospectiva de fuente secundaria, utilizando la base de datos de la consulta de cardiología del Hospital Pediátrico Dr. Hugo Mendoza entre enero 2017 y agosto 2019.

Resultados: el rango de edad de los pacientes: menor a 1 mes $40.7 \%$; 1 mes a 1 año $18 \%$; $1-4$ años $23.4 \%$; 5-9 ańos $7.6 \%$; $10-14$ ańos $7.6 \%$; mayor a 15 ańos $2.8 \%$. Las

\footnotetext{
a Médico Pediatra, Investigador Adjunto Centro de Investigación Dr. Hugo Mendoza. Hospital Pediátrico Dr. Hugo Mendoza, Santo Domingo, República Dominicana. Correo-e: herreramorbanmd@gmail.com, ORCID: 0000-0002-6678-6064

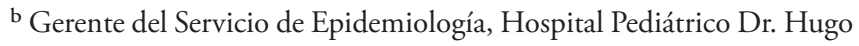
Mendoza, República Dominicana. Correo-e: manuel.colome@hhm.gob.do ORCID: 0000-0002-4562-6491
}

\begin{abstract}
Introduction: Congenital heart disease (CHD) is a group of pathologies where the structure or morphology of the heart is affected, that is, it occurs a disorder in the function of the organ, originated during the formation or embryonic cardiovascular development. CC represent the most common congenital malformation in children worldwide, with a prevalence of 4-14 per 1000 live births, with an increase in Asian countries in 13-16/ 1000 live births. ${ }^{1-4,6}$

Material and Methods: A descriptive and cross-sectional study was carried out, with retrospective data collection from secondary sources, using the cardiology consultation database of the Dr. Hugo Mendoza Pediatric Hospital between January 2017 and August 2019.

Results: The age range of the patients: less than 1 month $40.7 \%$; 1 month to 1 year $18 \%$; $1-4$ years $23.4 \%$; $5-9$ years $7.6 \%$; $10-14$ years $7.6 \%$; over 15 years $2.8 \%$. Heart disease affected both sexes interchangeably. According

\footnotetext{
* Investigador Adjunto Centro de Investigación Dr. Hugo Mendoza. Hospital Pediátrico Dr. Hugo Mendoza, República Dominicana.

${ }^{c}$ Correo-e: rayneidamendez@gmail.com, ORCID: 0000-0002-3720-0474

d Correo-e: 100208719ztf@gmail.com, ORCID: 0000-0003-0208-2743

${ }^{\mathrm{f}}$ Correo-e: cossetyhenriquez@gmail.com

g Correo-e: tatianame73@gmail.com

${ }^{\text {h }}$ Correo-e: yessi_alcantara@hotmail.com, ORCID: 0000-0002-3491-9897
} 
cardiopatías afectaron, indistintamente, a ambos sexos. De acuerdo con el tipo de cardiopatía la Acianógena con $92.1 \%$, y la Cianógena con $7.9 \%$.

Conclusiones: durante el periodo de estudio, hubo diferencias significativas entre la cantidad de casos recibidos anualmente en este centro, de 8/1000, 1/1000, 6/1000 pacientes atendidos para el 2017, 2018, 2019, respectivamente, deduciéndose que no hay una alta prevalencia anual para pacientes con este tipo de enfermedades en este centro.

Palabras clave: cardiopatía; tetralogía de Fallot; defectos del tabique interatrial; defectos del tabique interventricular.

\section{Introducción}

De acuerdo a la Organización Mundial de la Salud, las malformaciones congénitas representan una causa importante de mortalidad infantil, enfermedad crónica y discapacidad en muchos países. Se estima que 303,000 recién nacidos mueren dentro de las primeras cuatro horas de vida cada año, como consecuencia de malformaciones congénitas. Afectan 1 de cada 33 lactantes y causan 3,2 millones de discapacidades al año, lo que representa un gran indicador no solo para la salud pública global, sino también para las familias, comunidades, y sociedad que representa ese paciente. ${ }^{1}$

Las cardiopatías congénitas (CC) son un colectivo de patologías donde la estructura o morfología del corazón se ve afectada, lesionada o alterada, asimismo influye o interviene en la modificación, variación y perturbación de la fisiología del corazón, es decir, se produce un trastorno en la función del órgano, originado durante la formación o desarrollo cardiovascular embrionario. ${ }^{2}$ Las CC representan la malformación congénita más frecuente en niños a nivel mundial, con una prevalencia de 4-14 por 1000 nacidos vivos, destacándose un aumento en los países asiáticos en 13-16/1000 nacidos vivos. ${ }^{3-6}$

Diversos autores han observado y analizado que las CC tienen una prevalencia del $8 \%$ de los recién nacidos vivos al año y del $12.5 \%$ a los 16 años. ${ }^{7} \mathrm{La}$ to the type of heart disease, the Cornflower with $92.1 \%$, and the Cyanogen with 7.9\%.

Conclusions: During the study period, there were significant differences between the number of cases received annually in this center, of $8 / 1000,1 / 1000,6 / 1000$ patients treated for 2017, 2018, 2019 respectively, reducing that there is no high annual prevalence for patients with this type of diseases in this center.

Keywords: Heart disease; tetralogy of Fallot; heart septal defects.

mayoría de las causas son desconocidas, aunque se han asociado factores de riesgo maternos y ambientales como son: fumar, medicamentos, síndromes genéticos, aborto o enfermedades durante el embarazo. ${ }^{6-11} \mathrm{La}$ combinación de factores concomitantes asociados a la entidad, como consumo de alcohol, edad materna avanzada, uso de anticonceptivos y enfermedades maternas infecciosas en el primer trimestre de embarazo, aumentan el riesgo de CC. ${ }^{8}$

Ha sido considerado que varios defectos cardíacos son transmitidos a través de genes, cuyos alelos son alterados de forma dominante, por lo que se transfieren al poseer una copia que exprese la enfermedad. Esto significa que tanto hombres como mujeres son igualmente afectados. ${ }^{?}$

Las CC acianógenas son las más frecuentes y de menor severidad, entre las que se destacan los defectos del septo atrial (ASD) y ventricular (VSD) y la persistencia del conducto arterioso (PCA), variando ampliamente entre países. Por otra parte, las CC en menor grado las cardiopatías congénitas cianógenas son las de mayor complejidad, como la tetralogía de Fallot (TOF) y la transposición de grandes vasos (TGV). ${ }^{3-6}$

Estas enfermedades pueden no presentar signos ni síntomas. En algunas estos se ven en etapas más avanzadas de la vida. Cuando presentan síntomas los más frecuentes que podemos ver son: cianosis, 


\section{Epidemiología de cardiopatías congénitas en un hospital de tercer nivel, Santo Domingo Norte, República Dominicana}

arritmias, cansancio tras actividad física (en los bebés esto se ve como cansancio al alimentarse), dificultad respiratoria, entre otros ${ }^{10}$.

Aproximadamente el $3 \%$ de los recién nacidos con CC fallece durante el primer mes de vida, y entre los sobrevivientes un $25 \%$ puede requerir una intervención quirúrgica en el primer año de vida, aumentando el gasto en salud. , $^{6,11-13}$ La mortalidad asociada a CC depende de la severidad de la cardiopatía siendo reportada en un $12 \%$ de manera global, predominando las muertes en los primeros cinco años de vida. ${ }^{3}$ La determinación de la prevalencia de las CC depende del sitio de recolección de datos y el momento del estudio ecocardiográfico afectando la incidencia de CC de cierre espontáneo prematuro y los casos severos fallecidos. ${ }^{15}$

\section{Material y Métodos}

Se realizó un estudio descriptivo y transversal, con recolección de datos retrospectiva de fuente secundaria, utilizando la base de datos de la consulta de cardiología del Hospital Pediátrico Dr. Hugo Mendoza entre enero 2017 y agosto 2019. Se consideró la fecha de entrada más antigua como fecha de ingreso al sistema de salud.

La búsqueda incluyó los términos de insuficiencia/ estenosis mitral, insuficiencia/estenosis aórtica, insuficiencia/estenosis tricuspídea, insuficiencia/ estenosis pulmonar, persistencia del conducto arterioso, foramen oval permeable, comunicación o defecto del tabique auricular, comunicación o defecto del tabique ventricular, ventrículo único para las cardiopatías congénitas acianógenas, tetralogía de Fallot, transposición de grandes vasos, drenaje venoso anómalo, tronco arterioso común, atresia tricuspídea, atresia pulmonar para las cardiopatías cianógenas para recolectar la totalidad de pacientes con cardiopatías congénitas cianógenas o acianógenas que acudieron al centro de salud durante el periodo en estudio.
Una vez establecidos los criterios de inclusión se procedió a eliminar los registros duplicados depurando así la base de datos. Los datos fueron descritos en tiempo (frecuencia) y persona (edad, sexo y tipo de cardiopatía). Se excluyeron del análisis aquellos registros con datos incompletos de las variables descritas anteriormente. La prevalencia se calculó en torno a la relación de los casos evidenciados con la población atendida del centro de salud. Los datos se expresaron en medidas de frecuencia simple y de dispersión utilizando el programa SPSS en su versión 23.

\section{Resultados}

La frecuencia fue igual en ambos sexos, con un total de 284 pacientes $(50 \%)$. Según el rango de edad: menor a 1 mes con 231 pacientes ( $40.7 \%) ; 1$ mes a 1 año con 102 pacientes (18\%); $1-4$ años con 133 pacientes $(23.4 \%)$; 5-9 años con 43 pacientes (7.6\%); 10-14 años con 43 pacientes (7.6\%); mayor a 15 años con 16 pacientes (2.8\%). De acuerdo al tipo de cardiopatía la acianógena con 523 pacientes (92.1\%), y la cianógena con 45 pacientes (7.9\%). Distribución de las cardiopatías congénitas según el año: en el 2017 se diagnosticaron 247 pacientes (43.5\%), en el 2018 un total de 36 pacientes $(6.3 \%)$ mientras que en el 2019 con un total de 285 pacientes $(50.2 \%)$ (ver tabla 1$)$.

El diagnóstico más frecuente fue el defecto del tabique auricular con 306 pacientes (53.9\%), seguido del defecto del tabique ventricular con 125 pacientes (22\%), tetralogía de Fallot con 34 pacientes (6\%), conducto arterioso persistente con 32 pacientes (5.6\%), insuficiencia mitral congénita con 25 pacientes $(4.4 \%)$, estenosis de la válvula aórtica con 17 pacientes $(3 \%)$, y atresia de la arteria pulmonar con 9 pacientes (1.6\%) (ver tabla 2$)$.

El tipo de la cardiopatía congénita más frecuente en estos tres años de estudio correspondió a la de tipo acianógena. El diagnóstico de la cardiopatía congénita más frecuente según año, en el 2017: defecto del tabique auricular con 138 pacientes 
seguidos del defecto del tabique ventricular con 68 pacientes, tetralogía de Fallot con 14 pacientes, conducto arterioso persistente 11 pacientes. En el 2018: tetralogía de Fallot con 12 pacientes seguidos de la insuficiencia mitral congénita con 9 pacientes, atresia de la arteria pulmonar 5 pacientes, defecto del tabique ventricular con 4 pacientes. En el 2019: defecto del tabique auricular con 167 pacientes seguidos del defecto del tabique ventricular con 53 pacientes, conducto arterioso persistente 21 pacientes, estenosis de la válvula pulmonar 16 pacientes (ver tabla 3 ).

Tabla 1. Distribución de las cardiopatías congénitas según grupo etario $(\mathrm{n}=568)$

\begin{tabular}{|c|c|c|}
\hline & Frecuencia & Porcentaje \\
\hline \multicolumn{3}{|l|}{ Sexo } \\
\hline Femenino & 284 & 50 \\
\hline Masculino & 284 & 50 \\
\hline Total & 568 & 100 \\
\hline \multicolumn{3}{|l|}{ Rango De Edad } \\
\hline Menor A 1 Mes & 231 & 40.7 \\
\hline Entre 1 Mes - 1 Año & 102 & 18 \\
\hline Entre $1-4$ & 133 & 23.4 \\
\hline Entre 5-9 & 43 & 7.6 \\
\hline Entre $10-14$ & 43 & 7.6 \\
\hline Mayor A 15 & 16 & 2.8 \\
\hline Total & 568 & 100 \\
\hline \multicolumn{3}{|l|}{ Tipo de Cardiopatía } \\
\hline Acianógena & 523 & 92.1 \\
\hline Cianógena & 45 & 7.9 \\
\hline Total & 568 & 100 \\
\hline \multicolumn{3}{|l|}{ Año } \\
\hline 2017 & 247 & 43.5 \\
\hline 2018 & 36 & 6.3 \\
\hline 2019 & 285 & 50.2 \\
\hline Total & 568 & 100 \\
\hline
\end{tabular}

Fuente: elaborada por el equipo de investigación a partir de la información obtenida de la base de datos de la consulta del HPHM.
Tabla 2. Diagnósticos de las cardiopatías congénitas $(n=568)$

\section{Diagnóstico Principal}

Total Porcentaje

Atresia de la arteria pulmonar $\quad 9 \quad 1.6$

Coartación de la aorta

4

0.7

Conducto arterioso

persistente

Corazón triauricular

$32 \quad 5.6$

$1 \quad 0.2$

Defecto del tabique auricular $306 \quad 53.9$

Defecto del tabique

auriculoventricular

Defecto del tabique

ventricular

Estenosis de la válvula aórtica

Estenosis de la válvula mitral

1

0.4

Estenosis de la válvula

pulmonar

17

0.2

Foramen oval permeable

$$
4
$$

Insuficiencia de la válvula

aórtica

2

0.4

Insuficiencia de la válvula

pulmonar

$1 \quad 0.2$

Insuficiencia mitral congénita $25 \quad 4.4$

$\begin{array}{lll}\text { Insuficiencia tricúspide } & 1 & 0.2\end{array}$

Tetralogía de Fallot

34

6

Transposición de grandes

\begin{tabular}{lcc} 
vasos & 1 & 0.2 \\
Tronco arterioso común & 1 & 0.2 \\
\hline Total & 568 & 100 \\
\hline
\end{tabular}

Fuente: elaborada por el equipo de investigación a partir de la información obtenida de la base de datos de la consulta del HPHM. 
Tabla 3. Tipo de cardiopatía según el periodo $(\mathrm{n}=568)$

\begin{tabular}{|c|c|c|c|c|}
\hline \multicolumn{5}{|c|}{ Tipo de la cardiopatía congénita según año } \\
\hline Año & 2017 & 2018 & 2019 & Total \\
\hline Acianógena & 232 & 19 & 272 & 523 \\
\hline Cianógena & 15 & 17 & 13 & 45 \\
\hline Total & 247 & 36 & 285 & 568 \\
\hline \multicolumn{5}{|c|}{ Diagnóstico de la cardiopatía congénita según año } \\
\hline $\begin{array}{l}\text { Atresia de la arteria } \\
\text { pulmonar }\end{array}$ & 0 & 5 & 4 & 9 \\
\hline Coartación de la aorta & 0 & 2 & 2 & 4 \\
\hline $\begin{array}{l}\text { Conducto arterioso } \\
\text { persistente }\end{array}$ & 11 & 0 & 21 & 32 \\
\hline Corazón triauricular & 0 & 0 & 1 & 1 \\
\hline Defecto del tabique auricular & 138 & 1 & 167 & 306 \\
\hline $\begin{array}{l}\text { Defecto del tabique } \\
\text { auriculoventricular }\end{array}$ & 0 & 0 & 2 & 2 \\
\hline $\begin{array}{l}\text { Defecto del tabique } \\
\text { ventricular }\end{array}$ & 68 & 4 & 53 & 125 \\
\hline Estenosis de la válvula aórtica & 0 & 1 & 1 & 2 \\
\hline Estenosis de la válvula mitral & 0 & 1 & 0 & 1 \\
\hline $\begin{array}{l}\text { Estenosis de la válvula } \\
\text { pulmonar }\end{array}$ & 0 & 1 & 16 & 17 \\
\hline Foramen oval permeable & 4 & 0 & 0 & 4 \\
\hline $\begin{array}{l}\text { Insuficiencia de la válvula } \\
\text { aórtica }\end{array}$ & 0 & 0 & 2 & 2 \\
\hline $\begin{array}{l}\text { Insuficiencia de la válvula } \\
\text { pulmonar }\end{array}$ & 0 & 0 & 1 & 1 \\
\hline $\begin{array}{l}\text { Insuficiencia mitral } \\
\text { congénita }\end{array}$ & 10 & 9 & 6 & 25 \\
\hline Insuficiencia tricúspide & 1 & 0 & 0 & 1 \\
\hline Tetralogía de Fallot & 14 & 12 & 8 & 34 \\
\hline $\begin{array}{l}\text { Transposición de grandes } \\
\text { vasos }\end{array}$ & 1 & 0 & 0 & 1 \\
\hline Tronco arterioso común & 0 & 0 & 1 & 1 \\
\hline Total & 247 & 36 & 285 & 568 \\
\hline
\end{tabular}

Fuente: elaborada por el equipo de investigación a partir de la información obtenida de la base de datos de la consulta del HPHM.
Gráfico 1. Distribución temporal de las cardiopatías congénitas $(\mathrm{n}=568)$

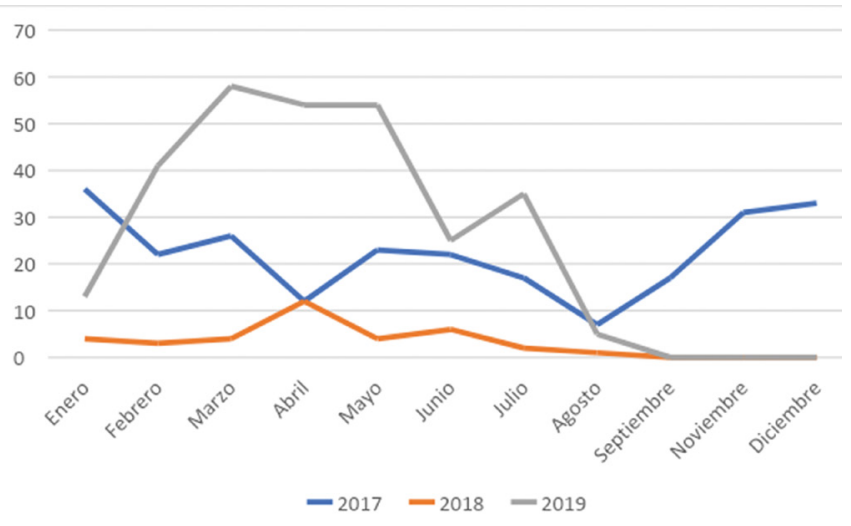

Fuente: elaborada por el equipo de investigación a partir de la información obtenida de la base de datos de la consulta del HPHM.

\section{Discusión}

La prevalencia de CC ha sido objeto de estudio de muchos países en la población neonatal debido a la existencia de CC que cierran tempranamente como el PCA o la muerte de los neonatos con CC Complejas; ${ }^{15}$ el comportamiento epidemiológico se ve modificado por características socio-económicas y antecedentes mórbidos de la madre ${ }^{6,11}$ razón por la cual durante el periodo neonatal la prevalencia difiere, evidenciándose en Noruega en 13.7/1000 nacidos vivos y decreciendo a $12.3 \%$ cuando se excluye el PCA del análisis, ${ }^{15}$ en el norte de la India en 8/1000 recién nacidos vivos, ${ }^{16}$ en Europa en 8/1000 nacidos, mientas que en Asia se estima en 9/1000 nacidos vivos, siendo la región del mundo con mayor prevalencia de CC, aun tomando en consideración las limitaciones concernientes a ser un estudio en un centro de salud pediátrico terciario donde no se reciben nacimientos, evidenciamos una prevalencia de CC fue de 247, 36, 285 pacientes atendidos para el 2017, 2018, 2019 respectivamente; pudiendo afectarse la prevalencia en los periodos estudiados por la migración de pacientes y las defunciones. 
El comportamiento epidemiológico de las CC evidenciadas en nuestro estudio difiere a lo publicado globalmente, donde predominan, al mostrar un comportamiento homogéneo entre ambos sexos. Esto podría deberse a características sociodemográficas de nuestra población; sin embargo, similar a otros estudios, predominan las CC Acianógenas menos severas como los defectos del septo atrial (ASD) y/o septo ventricular (VSD) y persistencia del conducto arterioso (PCA), en comparación con otras cardiopatías Cianógenas más complejas. Similar a otros estudios la CCC más frecuente fue la TOF. $3-6,11,15-18$

Factores como la etnia y situación socioeconómica pueden servir de condicionantes para el aumento de la prevalencia durante el 2017 en nuestro centro de salud, sin embargo, la población de descendencia afroamericana tiene menor riesgo de presentar CC en comparación con otras, ${ }^{17}$ razón por la cual la etnia de nuestra población puede servir de factor protector para el desarrollo de CC, siendo opuesto a los datos encontrados por nuestro estudio.

Aunque el descenso tan acentuado durante el 2018 carece de una explicación hasta el momento, podríamos suponer que la mortalidad durante el periodo neonatal en $8 \%$ aunado a una sobrevida a los 5 años de CC de un $76,{ }^{5}$ pueden disminuir la prevalencia de CC en este estudio. Nuestro país, al presentar mayores limitantes socioeconómicas, ausencia de un programa de detección de CC durante el periodo neonatal, tiene una menor sobrevida, lo que incrementa drásticamente la mortalidad de los pacientes con CC pudiendo causar períodos con picos y bajos de la prevalencia de la CC, como evidenciamos.

No obstante, se evidenció un aumento exponencial durante el 2019, comparado al 2018, siendo similar al estudio realizado por Moreta y Megías en el Hospital Robert Reid Cabral, donde se evidencia un aumento en los casos de cardiopatías congénitas, principalmente debido al consumo de alcohol durante el embarazo, con la idea de "limpiar a sus criaturas"13. Lo cual deja en claro, que la ignorancia sigue siendo un muro que evita que se refuercen las estrategias de prevención y orientación durante el embarazo, en nuestra sociedad.

\section{Conclusión}

Podemos resumir que, durante el periodo de estudio, hubo diferencias significativas entre la cantidad de casos recibidos anualmente en este centro, de 247 , 36, 285 pacientes atendidos para el 2017, 2018, 2019, respectivamente, deduciéndose así que no hay una alta prevalencia anual fija para pacientes con este tipo de enfermedades en este centro.

\section{Limitaciones}

Debido a las características metodológicas de la investigación pueden existir individuos con los diagnósticos estudiados que no recibieron atención en nuestro centro de salud, pudiendo influir en la prevalencia de la patología, lo cual incluye la posibilidad de individuos con los diagnósticos previos que estén recibiendo asistencia en otro centro de salud, o haber fallecidos durante el periodo neonatal.

\section{Conflicto de interés}

No se declara conflicto de interés para la realización de este estudio.

\section{Financiamiento}

No se recibió financiamiento para la realización de este estudio. 


\section{Referencias}

1. Organización Mundial de la Salud (OMS). Anormalidades congénitas. facts sheet No. 370. Septiembre 2016. Disponible en: http://www. who.int./mediacentre/factsheets/fs370/en/ index.html (inglés).

2. Otaigbe BE, Tabansi PN. Congenital heart disease in the Niger Delta Region of Nigeria: a four-year prospective echocardiographic analysis. Cardiovasc J Afr. 2014;25(6):265-8. Doi: 10.5830/CVJA-2014-055

3. Yeh SJ, Chen HC, Lu CW, Wang JK, Huang LM, Huang SC, Huang SK, Wu MH. Prevalence, Mortality, and the Disease Burden of Pediatric Congenital Heart Disease in Taiwan. Pediatrics \& Neonatology. 2013;54(2):113-8. Available from: http://dx.doi.org/10.1016/j. pedneo.2012.11.010

4. Sun PF, Ding GC, Zhang MY, He SN, Gao Y, Wang JH. Prevalence of Congenital Heart Disease among Infants from 2012 to 2014 in Langfang, China. Chin Med J. 2017;130:1069-73.

5. Yeh SJ, Chen HC, Lu CW, Wang JK, Huang LM, Huang SC, Huang SK, Wu MH. National database study of survival of pediatric congenital heart disease patients in Taiwan. Journal of the Formosan Medical Association. 2015;114(2): 159-163 Available from: https://doi.org/10. 1016/j.jfma.2012.10.006

6. Liu F, Yang Y-N, Xie X, Li X-M, Ma X, Fu ZY, et al. Prevalence of Congenital Heart Disease in Xinjiang Multi-Ethnic Region of China. PLoS ONE.2015;10(8):e0133961. Doi:10.1371/journal. pone. 0133961
7. Granado M. Epidemiología de las cardiopatías congénitas. (1 ra ed.). Capítulo 2 Epidemiología de las cardiopatías congénitas Lima: Víctor López Guzmán; 2017. Disponible en: https:// guzlop-editoras.com/web_des/med01/pediatria/cardio/pld4067.pdf

8. Alcántara YP, Reyes S. Factores de riesgo asociados a cardiopatías congénitas en pacientes menores de un año en el Hospital Infantil Dr Robert Reid Cabral. Revista Médica Dominicana. 2018;79(2):133-2.

9. Stanford Children's Health. Stanfordchildrens. org. 2020. [Online]. Disponible en: https:// www.stanfordchildrens.org/es/topic/default? $\mathrm{id}=$ factorscontributingtocongenitalheartdisease-90-P04891 [citado el 18 de enero 2020].

10. Mayo Clinic. (2019). Enfermedad cardíaca congénita en adultos. [Online] Disponible en: https://www.mayoclinic.org/es-es/diseases-conditions/adult-congenital-heart-disease/symptoms-causes/syc-20355456

11. Saxena, A. Congenital heart disease in India: A status report. Indian J Pediatr. 2005;55:107582. Available from: https://doi.org/10.1007/ BF02724185

12. Pinto Júnior VC, Branco KM, Cavalcante RC, et al. Epidemiology of congenital heart disease in Brazil. Rev Bras Cir Cardiovasc. 2015;30(2):219-24. Doi:10.5935/1678-9741. 20150018

13. Ministerio de Salud Pública. Estudio muestra alta incidencia cardiopatías congénitas en recién nacidos por consumo de alcohol en el embarazo - Ministerio de Salud Pública [Online]. 2019. Available from: https://www.msp.gob.do/web/? $\mathrm{p}=4949$ [cited 18 January 2020]. 
14. Bhardwaj R, Kandoria A, Marwah R, Vaidya P, Singh B, Dhiman P, Sood A, Sharma A. Prevalence of congenital heart disease in rural population of Himachal - A population-based study. Indian Heart Journal. 68(1):48-51. Available from: https://doi.org/10.1016/j.ihj.2015.08.022

15. Leirgul E, Fomina T, Brodwall K, Greve G, Holmstrøm H, Vollset SE, et al. Birth prevalence of congenital heart defects in Norway 1994-2009-A nationwide study. American Heart Journal. 2014;168(6):956-64.

16. Saxena A, Mehta A, Sharma M, Salhan S, Kalaivani $M$, Ramakrishnan $S$, et al. Birth prevalence of congenital heart disease: A cross-sectional observational study from North India. Ann Pediatr Card. 2016;9(3):205.
17. Van der Linde D, Konings EEM, Slager MA, Witsenburg M, Helbing WA, Takkenberg JJM, et al. Birth Prevalence of Congenital Heart Disease Worldwide. Journal of the American College of Cardiology. 2011;58(21):2241-7.

18. Bibi S, Gilani SYH, Bibi S. Spectrum of congenital heart disease in full term neonates. J Ayub Med Coll Abbottabad. 2018;30(1):67-70. Available from: https://www.ncbi.nlm.nih.gov/ pubmed/29504333 